\title{
A Stochastic Approach for The One-Dimensional Bin-Packing Problems
}

\author{
Cheng-Yan Kao \\ Department of Computer Science and Information Engineering, \\ National Taiwan University, Taipei, Taiwan \\ Feng-Tse Lin \\ Department of Applied Mathematics, Chinese Culture University, \\ Yangminshan, Taipei, Taiwan
}

\begin{abstract}
This paper presents a new stochastic approach called annealing-genetic algorithm for the one-dimensional bin-packing problem. This approach incorporates genetic algorithms into simulated annealing to improve the performance of simulated annealing. The genetic approach to simulated annealing seems to facilitate the exhaustive and parallel treatment of the problem and to increase the probability of finding global minimums. The empirical results show that the solution quality of this approach is better than or equal to the FFD (First-Fit-Decreasing) in the average cases but is better than the FFD in all the known worst cases. Unlike the FFD, no non-monotone anomaly have been found in our approach.
\end{abstract}

\section{INTRODUCTION}

The one-dimensional bin-packing problem is defined as follows. We are given a list of $\mathbf{n}$ weights and a set of bins each with equal capacity. The objective is to find a feasible assignment of all weights to bins that minimizes the total number of bins used. The bin-packing problem models several practical problems in such diverse areas as industrial control, computer systems, machine scheduling, VLSI chip layout, and etc. Some typical examples[3,12] are packing trucks with a given weight limit, one-dimensional cutting stock, cable-length optimization, assignment of segments of tracks on disks, prepaging, packing of variable length strings into fixed length words, and minimizing the number of processors necessary for completing all tasks by a given deadline[4]. From an engineering point of view, the problems are to make efficient use of time and space.

The bin-packing problem is NP-hard[9] so that a polynomial time algorithm for finding its optimal solution

Manuscript received March 15, 1992. This work was supported in part by a grant from the National Science Council of the R.O.C. under Grant NSC-80-0408-E002-26. is very unlikely to exist. Thus, several heuristics have been proposed over the past two decades. They are categorized either into on-line or off-line algorithms. An on-line algorithm is that each time a decision to be made to put a weight in a bin is done locally without looking at other weights appearing later on the list. The most popular and useful heuristics are Next-Fit (NF), First-Fit (FF), Best-Fit (BF), First-Fit-Decreasing (FFD), and Best-Fit-Decreasing (BFD). Both the FFD and the BFD are off-line algorithms, and the others are on-line algorithms. Among these algorithms, the FFD is the most prominent one. Let $N F(L)$ be the number of bins used by the NF algorithm executed on the list $L$. The performance measure $[12,21]$ is usually computed as the ratio of $N F(L)$ to the optimum number of bins $L^{*}$. Then, the maximum of the ratio

$$
\mathrm{R}_{\mathrm{NF}}(\mathrm{k})=\frac{\mathrm{NF}(\mathrm{L})}{\mathrm{L}^{*}}
$$

indicates the maximum deviation of the NF algorithm with $L^{*}=k$. The main results of the worst case behavior of these algorithms in literatures[11, 12] can be summarized as follows:

$$
\begin{aligned}
& \lim _{k \rightarrow \infty} R_{N F}(k)=2, \\
& \lim _{k \rightarrow \infty} R_{F F}(k)=1.7, \\
& \lim _{k \rightarrow \infty} R_{B F}(k)=1.7, \\
& \lim _{k \rightarrow \infty} R_{F F D}(k)=\frac{11}{9}, \\
& \lim _{k \rightarrow \infty} R_{B F D}(k)=\frac{11}{9} .
\end{aligned}
$$

However, the FFD has some disadvantages as follows.

(1) In some special cases, $\mathrm{BFD}(\mathrm{L}) \leq \mathrm{FFD}(\mathrm{L})$.

(2) They have some abnormal cases by giving a list $\mathrm{L}$ and a sublist $L^{\prime}, L^{\prime} \subseteq L$, that obtaining $F F D\left(L^{\prime}\right)>F F D(L)$.

(3) The algorithm is a constructive method so that it has no chance for further improvements in the worst cases. That is, the FFD has the worst case of $22 \%$ error performance which cannot be further improved. As far as we know, there is no bin-packing heuristic that does not have similar worst case and anomaly phenomenonas the FFD does. 
Our work is motivated by using a stochastic approach to overcome these shortcomings. In this paper, we propose a nature-based stochastic approach as a new approximation algorithm for solving the bin-packing problem and as a solution improving tool for the FFD in its worst cases. Our approach is called annealing-genetic algorithm which is incorporating genetic algorithms[10] into simulated annealing[17] to improve the performance of simualted annealing. Empirical results show that the quality of the solution of our approach is better than or equal to the FFD in the average cases but is better than the FFD in all the known worst cases. Moreover, our approach has no anomaly as the FFD does in our test data and always obtains better solutions than the BFD without any exceptions.

\section{THE ANNEALING-GENETIC APPROACH}

Since the FFD is a deterministic constructive algorithm, a stochastic approach has the potential of overcoming its shortcomings. Both simulated annealing and genetic algorithms are nature-based stochastic optimization techniques, the former is based on thermodynamics and the latter is based on natural evolution. The major advantages of nature-based algorithms are their broad applicability, flexibility, ease of implementation, and potential to find the optimal solutions $[6,18]$. However, their disadvantages are as follows. Simulated annealing may require a long computation time in order to converge to the optimal solutions. Moreover, without a carefully designed annealing schedule, the simulated annealing may easily be trapped into a local optimum. Genetic algorithms are not well suited to performing finely-tuned local search and having the possibility of premature convergence due to the lost alleles in a certain generation $[6,10]$. Also, we need to map the problem into special representation to be used by genetic algorithms.

The main ideas of our approach in speeding up simulated annealing are as follows. On the contrary to just one starting point and one ending point of a Markov chain in simulated annealing, there are many starting points and ending points to construct many search paths in our approach (see Fig. 2). The starting points are selected from the old generation $P_{k}$ based on their fitness values, i.e. cost function values, and the end points are placed in $P^{\prime} \mathbf{k}+\mathbf{1}$ become the candidates for the population of the new generation $\mathbf{P}_{\mathbf{k}+1}$. Next, we apply the genetic operators such as crossover, mutation, and inversion to $P^{\prime} k+1$ to obtain the new ending points placing in $\mathrm{P}_{\mathbf{k}+1}$ and these new ending points have less average cost than the old ending points in $P_{k+1}^{\prime}$. Finally, the new ending points become the population of the new generation (see Fig. 3 and Fig. 4). A detailed discussion of the genetic operators is given in section 5.3 .

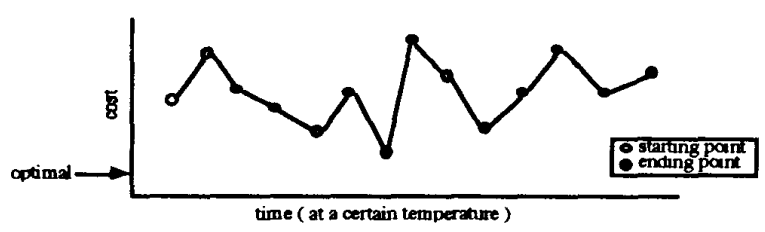

Fig. 1. A finite-length Markov chain is generated at a certain temperature with a single starting point and a single ending point by simulated annealing.

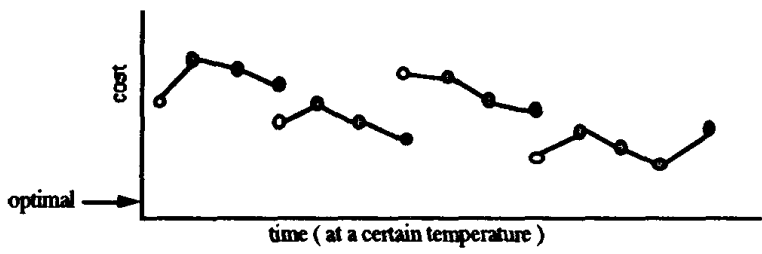

Fig. 2. There are many starting points and ending points to construct search paths by the annealing-genetic approach.

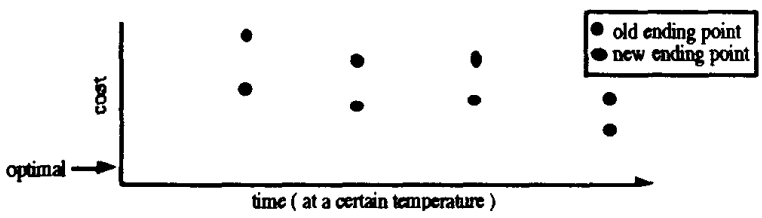

Fig. 3. By applying the genetic operators to obtain new ending points with lower costs than the old ones.

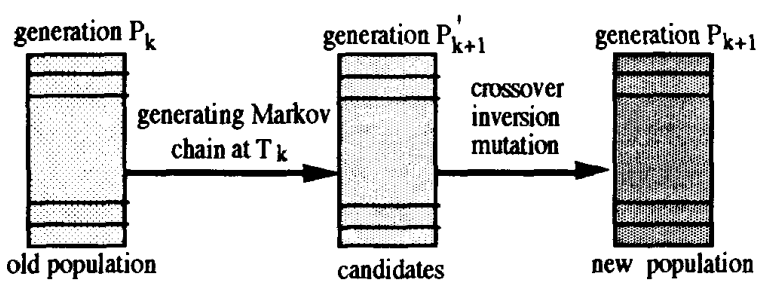

Fig. 4 The concept of the annealing-genetic approach.

The important parameters affecting the efficiency of simualted annealing are the initial temperature, the total length of Markov chains, the move generation strategy, and the frozen condition $[1,18]$. We use the concepts from genetic algorithms to control these parameters. The 
annealing-genetic algorithm starts with a randomly generated population $\mathrm{P}_{\mathrm{O}}^{\prime}$. Next, the genetic operators are applied to produce a new population $\mathrm{P}_{\mathrm{O}}$ by rejecting the higher cost offsprings so that the average cost of the population is less than the random one $\mathrm{P}_{\mathrm{o}}$. Then, the search paths are generated from the points of $P_{O}$ at a small value of temperature $T_{0}$ until the first generation $P_{1}$ is created. The initial value of the temperature $T_{1}$ is to be determined as follows. For the sake of the efficiency of the algorithm, we define the acceptance probability of a detrimental move to be 0.6. From Metropolis criterion Pr $=\exp \left(-\Delta C / T_{k}\right)$, where $\Delta C=$ new cost - old cost, we obtain $T_{k}=-\Delta C / \ln \operatorname{Pr}=-\Delta C / \ln 0.6 \approx 2 \Delta C$. The largest detrimental move in $P_{1}$ is defined as $\Delta C=$ (the highest cost - the lowest cost) / population_size. Thus, $\mathrm{T}_{1}$ is obtained by dividing the difference between the highest cost and the lowest cost of $P_{1}$ by half of the population size, i.e.

$\mathrm{T}_{1}=\frac{\text { the highest cost }- \text { the lowest cost }}{\text { population_size } / 2}$.

We let the lowest cost of $P_{1}$ to be the starting point and the annealing process is then initiated.

The total length of search paths at the temperature $T_{\mathbf{k}}$ is bounded by the population size, in particular, these paths are generated from multiple points of the population. The basic rules are as follows. Starting off with the point of lowest cost in the old generation, a next point is generated from the current point by the move generation strategy. If it is accepted by the Metropolis criterion, the next point not only becomes the current point but also as a member of the next generation; otherwise, a new starting point from the old generation is attempted and the point of lowest cost in the old generation is reproduced as a member of the next generation. The process is continued until the new population is generated. The point of lowest cost in each generation is copied to an independent solution vector as the search continues and is printed at the end of the run. Finally, when $80 \%$ of the population in a certain generation has the same cost as the solution vector, the frozen condition is signalled. After performing a local search procedure, we stop the algorithm.

\section{EXPERIMENTAL RESULTS}

In this section we provide experimental results to demonstrate the effectiveness of the proposed annealing-genetic $(\mathrm{AG})$ algorithm. We make the following assumptions in the experiments :
(1) The bins capacity is 1000 .

(2) The weights of the list are given randomly in $[1,999]$.

The first experiment concerns with the performance analysis of the average behavior of the AG algorithm and the other well-known heuristics. The list sizes are from 10 to 5120. One hundred sets of test data are run for each list size and the average number of bins is computed. Table I shows the results of the first experiment. The AG algorithm has the best average performance among all the testing algorithms. The second experiment concerns the well-known worst case of the FFD and BFD algorithms which have $F F D(L) / L^{*}=B F D(L) / L^{*}=11 / 9$ [12]. The first list is $L_{1}=\{250-2 \varepsilon(x 12), 250+2(x 6), 250+\varepsilon(x 6)$, $500+\varepsilon(x 6)\}$, where $\varepsilon$ is increasing from 1 to 30 and $250+2(\times 6)$ means 6 copies of $250+2$. The results of the average number of bins used are shown in Table II, where $A G\left(L_{1}\right)=9$ while $F F D\left(L_{1}\right)=B F D\left(L_{1}\right)=11$. The second list is $L_{2}=\{500-2 \varepsilon(\times 18), 333+\varepsilon(\times 18), 167+\varepsilon(\times 18)\}$, where $\varepsilon$ is increasing from 1 to 83 . The results are also shown in Table II, where $A G\left(L_{2}\right)=18, F F D\left(L_{2}\right)=20$, and $B F D\left(L_{2}\right)=20.6$. In the third experiment we deal with the special case of $B F D(L)<F F D(L)$ and $B F D(L)=L^{*}$. The list is $L_{3}=\{600+2 \varepsilon(x 5), 400(x 10), 200(x 5), 200-\varepsilon(x 10)\}$, where $1 \leq \varepsilon \leq 99$. This list has the feature of FFD(L) / $\mathrm{BFD}(\mathrm{L})=11 / 10$. However, the AG algorithm also obtains the optimal solution $A G(L)=10$. The fourth experiment concerns with the well-known anomaly of FFD. The bins capacity is 396 in this case. The empirical result shows that $A G\left(L_{4}\right)=A G\left(L_{4}\right)=12$ without FFD's anomaly ( also see Table II ). The last experiment concerns with the best case of FFD algorithm which has FFD(L) / $L^{*}=1$. Coffman, Garey and Johnson[5] have shown that suppose all item sizes are of the form $(1 / k) j, j \geq 0$, for some fixed positive integer $k$, then $F F D(L)=L^{*}$, i.e., FFD heuristic achieves the optimal packing in this class of problems. Table III shows the results for the experiment of AG against other heuristics in this class of FFD-favorable problems. The list $\mathrm{L}$ is of the form $(1 / 2) \mathrm{j}, 1 \leq \mathrm{j} \leq 4$. In this test case, $F F D(L)=F F(L)=A G(L)=L^{*}$.

The above experiments show that the solution quality of $\mathrm{AG}$ is equal to if not better than the FFD even in the most unfavorable test cases. It is a common phenomenon that certain new algorithms specifically designed for improving worst case behavior may be comparatively bad on the average. The $A G$ is exceptional to this rule. It is very robust. In [8], Frederickson gives a rigorous proof of the rather intuitive fact that, for uniformly distributed item size, the ratio FFD(L) / $L^{*}$ approaches 1 as the number of items approaches infinite. Karmarkar[14] has a result which implies that, for uniformly distributed item sizes, the ratio 
TABLE I

PERFORMANCE ANALYSIS OF RANDOMLY GENERATED TEST DATA

\begin{tabular}{|c|c|c|c|c|c|c|c|c|c|c|}
\hline \multirow{2}{*}{$\begin{array}{c}\text { List size } \\
\text { n }\end{array}$} & \multicolumn{6}{|c|}{ Average number of bins } & \multicolumn{4}{|c|}{$A G$} \\
\hline & NF & BF & FF & BFD & FFD & AG & $\begin{array}{c}\text { expected } \\
\text { waste } *\end{array}$ & $\begin{array}{r}\text { \%waste } \\
* *\end{array}$ & $\frac{\sqrt{\text { moves }}}{n}$ & $\frac{\sqrt{\text { time }}}{n}(\mathrm{sec})$ \\
\hline 10 & 7.1 & 6.4 & 6.4 & 6.2 & 6.2 & 6.2 & 1.2 & 24 & 2.135 & 0.192 \\
\hline 20 & 132 & 11.5 & 11.6 & 11.0 & 11.0 & 11.0 & 1.0 & 10 & 1.849 & 0.137 \\
\hline 40 & 26.4 & 22.5 & 22.8 & 21.7 & 21.7 & 21.7 & 1.7 & 8.5 & 1.749 & 0.135 \\
\hline 80 & 53.3 & 44.2 & 44.7 & 42.7 & 42.6 & 42.6 & 2.6 & 6.5 & 1.524 & 0.123 \\
\hline 160 & 106.0 & 86.0 & 87.1 & 82.8 & 82.7 & 82.7 & 2.7 & 3.38 & 1.425 & 0.116 \\
\hline 320 & 211.5 & 168.7 & 170.8 & 162.9 & 162.8 & 162.8 & 2.8 & 1.75 & 1.304 & 0.105 \\
\hline 640 & 424.5 & 333.8 & 337.8 & 324.1 & 323.8 & 323.6 & 3.6 & 1.13 & 1.284 & 0.103 \\
\hline 1280 & 849.0 & 662.2 & 670.1 & 646.5 & 645.9 & 645.6 & 5.6 & 0.88 & 1.165 & 0.102 \\
\hline 2560 & 1700.7 & 1313.6 & 1328.5 & 1287.8 & 1286.6 & 1286.1 & 6.1 & 0.47 & 1.165 & 0.102 \\
\hline 5120 & 3402.5 & 2615.6 & 2639.2 & 2573.7 & 2570.2 & 2569.7 & 9.7 & 0.38 & 1.141 & 0.100 \\
\hline
\end{tabular}

* The term "expected waste" refer to the average number of bins required in excess of the expected sum, $n / 2$.

** The term "\%waste" is the percentage of expected waste divided by $n / 2$.

+ Moves mean the average number of moves of 100 test data.

++ Time is the average execution time of 100 test data. The algorithm is implemented on a 80386-based PC written in Turbo-C.

TABLE II

RESULTS OF THE FFD AND THE BFD IN THE WORST CASES

\begin{tabular}{|c|c|c|c|c|c|c|}
\hline \multirow[b]{2}{*}{ Lists } & \multirow{2}{*}{ Capacity } & \multirow{2}{*}{ Instances } & \multicolumn{3}{|c|}{ Average number of bins } & \multirow{2}{*}{ Remarks } \\
\hline & & & AG & FFD & BFD & \\
\hline $\mathbf{L}_{1}$ & 1000 & 30 & $9^{*}$ & 11 & 11 & FFD(L) $/ L^{*}=11 / 9$ \\
\hline $\mathbf{L}_{2}$ & 1000 & 83 & $18^{*}$ & 20 & 20.6 & FFD(L) $/ L^{*}=10 / 9$ \\
\hline $1 / 3$ & 1000 & 99 & $10^{*}$ & 11 & $10^{*}$ & BFD $(L)<$ FFD $(L)$ \\
\hline L4. & 396 & 1 & 12 & 12 & 13 & \multirow{2}{*}{$F F D\left(L^{\prime}\right)>F F D(L)$} \\
\hline$L_{4}$ & 396 & 1 & 12 & 13 & 12 & \\
\hline
\end{tabular}

$L_{1}=\{250-2 \varepsilon(x 12), 250+2 \varepsilon(x 6), 250+\varepsilon(x 6), 500+\varepsilon(x 6)\}, 1 \leq \varepsilon \leq 30$.

$L_{2}=\{500-2 \varepsilon(x 18), 333+\varepsilon(x 18), 167+\varepsilon(x 18)\}, 1 \leq \varepsilon \leq 83$.

$\mathrm{L}_{3}=\{600+2 \varepsilon(\times 5), 400(\times 10), 200(\times 5), 200-\varepsilon(\times 10)\}, 1 \leq \varepsilon \leq 99$.

$\mathbf{L}_{4}=\{285,188(x 6), 126(x 18), 115(x 3), 112(x 3), 75,60,51,12(x 3), 10(x 6), 9(x 12)\}$.

$\mathrm{L}_{4}{ }^{\prime}=\mathrm{L}_{4}-\{75\}$.

* denotes the optimal solution. + the instances mean the number of test cases.

TABLE III

PERFORMANCE OF FFD-FA VORABLE PROBLEMS

\begin{tabular}{|r|r|r|r|r|r|r|}
\hline \multirow{2}{*}{$\begin{array}{c}\text { List size } \\
\mathbf{n}\end{array}$} & \multicolumn{6}{|c|}{ Number of bins needed } \\
\cline { 2 - 7 } & NF & BF & FF & BFD & FFD & AG \\
\hline 10 & 3 & 3 & 3 & 3 & 3 & 3 \\
\hline 20 & 6 & 6 & 5 & 5 & 5 & 5 \\
\hline 40 & 11 & 12 & 10 & 10 & 10 & 10 \\
\hline 80 & 23 & 21 & 19 & 20 & 19 & 19 \\
\hline 160 & 43 & 42 & 38 & 40 & 38 & 38 \\
\hline 320 & 86 & 83 & 75 & 80 & 75 & 75 \\
\hline 640 & 172 & 164 & 150 & 160 & 150 & 150 \\
\hline 1280 & 348 & 323 & 300 & 320 & 300 & 300 \\
\hline 2560 & 693 & 647 & 600 & 640 & 600 & 600 \\
\hline
\end{tabular}

$+\mathrm{L}=\{500(\mathrm{x} n / 4), 250(\mathrm{x} n / 4), 125(\mathrm{x} n / 4), 62.5(\mathrm{x} \mathrm{n} / 4)\}$ and the items of $L$ are placed in a randomly order. of $L^{*}$ to $n / 2$, the expected total of item sizes divided by the bin size, is 1 as the number of items approaches infinite. Therefore, for reasonably large uniformly distributed item sizes list, the approximation of $\mathrm{L}^{*}$ by $\mathrm{n} / 2$ is indeed a very good one and the average case performance of FFD is asymptotically optimal. The "expected waste" column of Table I refers to the average number of bins required in excess of the expected sum, $n / 2$. Johnson[12] has pointed out that the solution quality of the FFD and the BFD for the average case problems is about $2 \%$ error rate. His conclusion is based on a simulation run of 25 data sets of 200 -item list. As the "percent-waste" column of Table I shows, the error rate of AG, as well as FFD, is less than $0.5 \%$ for large item list. 


\section{DISCUSSIONS}

In response to two important questions raised by Johnson [12], Yao [21] has proposed two refined algorithms, the Refined First-Fit (RFF) and the Refined First-Fit-Decreasing (RFFD), which are strictly better than the FF and the FFD algorithms respectively. The questions are (1) Is there a polynomial on-line algorithm better than the FF? and (2) Is there any polynomial algorithm better than the FFD? The ratios of the RFF and the RFFD are as follows

$\lim _{k \rightarrow \infty} R_{R F F}(k)=\frac{5}{3}$, and

$\lim _{k \rightarrow \infty} R_{R F F D}(k) \leq \frac{11}{9}-\varepsilon$, for somefixed $\varepsilon>0$.

Coffman, Garey and Johnson[5] devised the Modified-First-Fit-Decreasing(MFFD) algorithm for which a tight asymptotic bound,

$\lim _{k \rightarrow \infty} R_{M F F D}(k)=\frac{71}{60}$

was proved. The algorithm is based on a careful analysis of the 11/9 examples for FFD and what causes FFD to mispack them. Nevertheless, the RFFD and the MFFD still have the same shortcomings as the FFD does. Most of the work on the one-dimensional bin packing problems in the seventies and early eighties has been concerned with approximation algorithms (denoted by $A$ ) of improved asymptotic bound

$\lim _{\mathbf{k} \rightarrow \infty} \mathbf{R}_{\mathrm{A}}(\mathbf{k})$.

Two major results of Fernandez de la Vega and Lueker[7] and Karmarkar and Karp[15] in early eighties represented a sharp departure from this line of research. Fernandez de la Vega and Lueker answer negatively to Yao's question of whether there exists a limit to the best asymptotic performance ratio for a polynomial algorithm[7]. They showed that techniques from the knapsack approximation scheme could be used in designing bin-packing algorithms satisfying

$A(L) \leq(1+\varepsilon) L^{*}+(1 / \varepsilon)^{2}$,

and having the running time linear in the length of $L$ for fixed $\varepsilon$ but growing exponentially in $(1 / \varepsilon)^{2}$. Karmarkar and Karp eliminated a shortcoming of the above result. They used techniques from complexity theory and interior-point polynomial time algorithm of linear programming to devise a fully polynomial approximation scheme, where the running time is a polynomial in both $1 / \varepsilon$ and the length of $\mathrm{L}$, and the additive constant is also a polynomial in $1 / \varepsilon$. A interesting results is that there exist polynomial time approximation algorithms with

$\lim _{\mathbf{k} \rightarrow \infty} R_{A}(k)=1$.

However, at present the above results are mainly theoretical in their significance, because the coefficients hidden in the term "polynomial time" are too large for practical purpose. For example, the time bound of Karmarkar and Karp algorithms including a term of $(1 / \varepsilon)^{16}$.

Our computational experience with the AG approach show that the solution quality is very good. In fact, it outperforms all the bin-packing heuristics we have tested in solution quality and it is very efficient in running time. By decreasing the control temperature in the annealing schedule more carefully and enlarging the population size, we can obtain a better quality solution. The AG algorithm has the feature of (1) it can be viewed as a simulated annealing algorithm with population-based transition and genetic operation-based quasi-equilibrium control, (2) it can be viewed as a genetic algorithm with Boltzmann-type selection operator. It is easy to see that the AG algorithm has a polynomial time complexity function because both finite Markov chain simulated annealing and genetic algorithm are polynomial time algorithms. In fact, the proposed AG approach can be applied to various NP-hard optimization problems, e.g., traveling salesman problems[19], knapsack problems, and set partition problems[13]. However, different problems may have to use different domain-specific knowledge. For the bin-packing problem, the cost function, the move generation strategy, and the genetic operators are to be specified using of the special structure of the bin-packing problem.

\section{A. The Cost Function}

We shall assume that every bin has capacity $\gamma$, and there are $n$ weights in the list, $0<a_{i} \leq \gamma$ for $1 \leq i \leq n$. The sum of $a_{i}$ packed in $a$ bin $B_{j}$ is called the content of $B_{j}$ and will be denoted by $W\left(B_{j}\right)$. We called $\gamma-W\left(B_{j}\right)$ the gap of $\mathrm{Bj}_{\mathrm{j}}$ and it is denoted by Gapj. Then, Gapj $=\gamma$ is an empty bin, $G_{a p}=0$ is a full bin, and $G a p_{j}<0$ is an illegal packing. A penalty cost will be incurred to $G_{a p}$ if there is illegal packing for $B_{j}$, and the penalty cost increases as the temperature decreases. That is,

$\operatorname{CosT}\left(B_{j}\right)=\left\{\begin{array}{l}G a p_{j}, \text { if } G a p_{j} \geq 0 . \\ \left(G a_{j}\right)^{2}+\text { penalty_cost }\left(T_{k}\right), \text { if } G a p_{j}<0 .\end{array}\right.$

The assignment vector $A[1: n]$ denotes all weights to bins where $A[i]=j$ meaning $a_{i}$ is assigned to $B_{j}$. Thus, the cost of an assignment $A$ is calculated by

$$
\cos T(A)=\sum_{f=1}^{m_{T}} \operatorname{cosT}\left(B_{j}\right) \text {. }
$$

\section{B. The Move Generation Strategy}

There are two strategies to be used in our algorithm for 
generating the next point of the search path. The first one is greedy move and the second is swapping move. By greedy move, a weight a is randomly selected from the bin, say $B_{1}$, which has the largest gap among all bins. Next, this weight is assigned to another bin, say $\mathrm{B}_{2}$, in an attempt to decrease the current number of bins needed. If $B_{2}$ has enough room for $a_{i}$, change $A[i]$ from 1 to 2 . By swapping move, we randomly choose two weights, say $a_{i}$ and $a_{j}$, which have been assigned to bins, say $B_{1}$ and $B_{2}$ respectively, and then exchanging their roles. That is, we change $A[i]=1$ and $A[j]=2$ to $A[i]=2$ and $A[j]=1$ respectively (see Fig. 5). In these moves, the change of costs is calculated by

$\Delta C-\left(\operatorname{cosT}\left(B_{1}^{\prime}\right)+\operatorname{cosT}\left(B_{2}^{\prime}\right)\right)-\left(\operatorname{cosT}\left(B_{1}\right)+\operatorname{cosT}\left(B_{2}\right)\right)$.
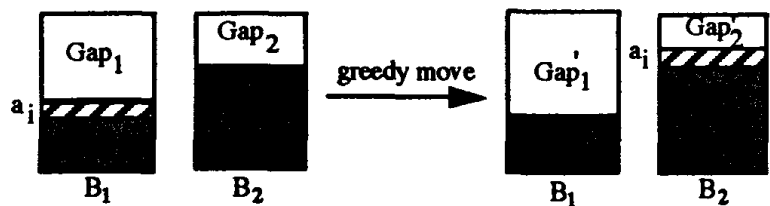

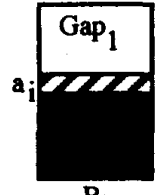

B1

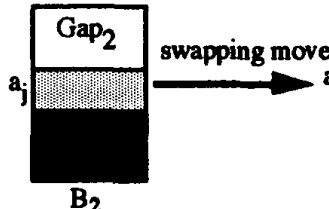

$\mathrm{B}_{2}$

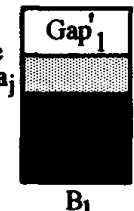

$\mathbf{B}_{1}$

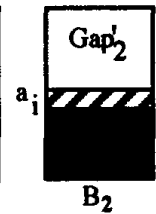

$\mathbf{B}_{2}$
Fig. 5. The move generation strategy.

\section{The Genetic Operators}

The three genetic operators not only modify the structure of the population to create new structures but also reduce the average cost of the population. However, no illegal packing are allowed. The crossover operator we used in the algorithm is the usual crossover operator in the genetic algorithm literature[6,16]. Crossover takes two selected parents, splits the structures at the same two randomly determined crossing sites, and then creates two offsprings by swapping the middle portion of the structures. Hence, the function of crossover is to generate rearrangements of coadapted groups of substructures from high performance structures. Nevertheless, crossover may produce the illegal packing offsprings. The second operator is inversion. Inversion alters the sequence between two randomly assigned indices in a single structure. This action modifies the configuration of weights to bins, providing a more fertile ground for reducing the total number of bins by the greedy move in simulated annealing. The final operator, mutation, is quite different from the usual mutation operator. This operator functions at a higher probability stead of lower probability as the usual genetic algorithms do $[6,10]$. The intention of using mutation operator in our approach is to ensure that all points in the search space remain reachable by simulated annealing. This can be easily done by changing randomly selected weights of bins in a single structure. However, mutation acts as a large randomly change of two bins by our approach. About half of the number of weights assigned in each bin are randomly selected and swapped. The genetic operators are performed in the following steps.

Step 1. The parents are selected from the population based on their fitness values. Then, the crossover operator is applied to produce their offsprings. The offsprings must have their costs less than the average cost of the old generation, otherwise, the parents continue the following steps.

Step 2. The inversion operator is applied to the parents for reordering their own sequence to obtain new costs. If the new cost is lower than the old one, the parent is copied to the next generation; otherwise, the parents continue the next operation.

Step 3. The mutation operator is applied to the parents expecting a lower cost. Finally, the parents are copied to the next generation.

These steps are repeated again and again until the population of the next generation is created.

\section{CONCLUSIONS}

We have presented an $\mathrm{AG}$ approach for solving the bin-packing problem. In conclusion, we would like to point out that our work generate the following important results for improving simulated annealing and for solving the one-dimensional bin-packing problem :

(1) There are multiple search states in a population instead of a single state, the probability of trapping into local minima is greatly reduced.

(2) The frozen condition of the system is the convergence of the population that seems more accurate and easier to detect than conventional simulated annealing approach.

(3) When applying our AG algorithm to the bin-packing problems, the empirical results show that the solution quality of our approach is better than or equal to the FFD in the average cases but is muc ${ }^{*}$ better than the FFD in all the known worst cases. Morcuver, unlike the FFD, no non-monotone anomaly has been found in our approach.

(4) The FFD family heuristics (i.e. FFD, BFD, FF, NF) are deterministic, solution construction heuristics. Our approach is a stochastic, solution improvement algorithm. Therefore, our approach can be used as an alternative, or as an improving tool for these FFD family heuristics by applying the $A G$ approach to the result of the heuristics as the initial population of our algorithm. 
(5) The AG approach for the bin-packing problems is empirically better then $O\left(n^{2}\right)$ as can be seen in Table I. Our computational experiments demonstrate that the $A G$ approach is empirically better than the FFD and the BFD. The error rate of the AG approach for the one-dimensional bin-packing problem is empirically less than $0.5 \%$ for large size problem.

The research activities of the bin-packing problem have been extended to the two-dimensional and the three-dimensional bin-packing problems in these couple years[20]. However, most heuristics have very poor worst case performance[20]. Our result can be easily extended to the multi-dimensional bin-packing problems. We believe that the performance of our approach for the multi-dimensional case would be better than most FFD-based heuristics. We will investigate this issue in the near future.

\section{REFERENCES}

[1] E.H.L. Aarts and J. Korst, Simulated annealing and Boltzmann machines, John Wiley and Sons publishing, 1989.

[2] J.L. Bentley, D.S. Johnson, F.T. Leighton, and C.C. McGeoch, "An experimental study of bin packing", Proceeding of Allerton Conference, University of Illinois, 1983.

[3] E.G. Coffman, (editor), Computer and job-shop schedule theory, John Wiley \& Sons Publishing, NewYork, 1976.

[4] E.G. Coffman, M.R. Garey, and D.S. Johnson, "An application of bin-packing to multiprocessor scheduling", SIAM Journal of Computing.1978, pp.1-17.

[5] E.G. Coffman, M.R. Garey, and D.S. Johnson, "Approximation algorithm for bin packing -- an updated survey", in analysis and design of algorithms in combinatorial optimization, edited by G. Ansiello and M. Lucertini, CISM courses and lectures \#266, Springer-Verlag, New York, 1981, pp.147-172.

[6] L. Davis, Genetic algorithms and Simulated annealing, Morgan Kaufmann Publishers, Inc., 1987.

[7] W. Fernandez de la Vega and G.S. Lueker, "Bin packing can be solved within $1+\varepsilon$ in linear timen ${ }^{n}$, Combinatorica 1, 1981, pp. 349-355.

[8] G.N. Frederickson, "Probabilistic analysis for simple one and two dimensional bin packing algorithms",
Information Processing Letters, 11, 1980, pp.156-161.

[9] M.R. Garey, and D.S. Johnson, Computers and Intractability : a guide to the Theory of NP-Completeness, Freeman, San Francisco, 1979.

[10]D.E. Goldberg, Genetic algorithms: In search, Optimization and Machine Learning, Addison-Wesiey Publishing Company, Reading Mass., 1989.

[11]D.S. Johnson, "Fast algorithms for bin-packing", Journal of Computer \& System Sciences 8, 1974. pp. $272-314$

[12] D.S. Johnson, A. Demers, J.D. Ullman, M.R. Garey, and R.L. Graham, "Worst case performance bounds for simple one-dimensional packing algorithm", SIAM Journal on Computing 3, 1974, pp. 299-326.

[13] C.Y. Kao and F.T. Lin, "An annealing-genetic approach for some NP-hard problems", accepted to Second International Symposium on Artificial Intelligence and Mathematics, Fort Landerdale, Florida, 1992.

[14]N. Karmarkar, "Probabilistic analysis of some bin packing problems", Proceeding of 23rd Annual Symposium on Foundations of Computer Science, 1982.

[15] N. Karmarkar, and R.M. Karp, "An efficient approximation scheme for the one-dimensional bin packing problem", Proceeding of 23rd Annual Symposium on Foundations of Computer Science. 1982, pp.312-320.

[16] A. Kenneth, De Jong, and W.H. Spears, "Using genetic algorithms to solve NP-Complete problems". Third International Conference on Genetic Algorithm, George Mason University, 1989, pp. 124 - 132.

[17]S. Kirkpatrick, C.D. Gelatt and M.P. Vecchi, "Optimization by simulated annealing", Science 220 , 1983, pp. 671-680.

[18] van P.J.M. Laarhoven and E.H.L. Aarts, Simulated annealing : theory and applications, D. Reidel Publishing Company, 1987.

[19]F.T. Lin, C.Y. Kao and C.C. Hsu, "Incorporating genetic algorithms into simulated annealing". The 4th International Symposium on Artificial Intelligence, Cancun, Mexico, Nov. 13-15, 1991. pp. 290-297.

[20] P.S. Parekh, Bin packing algorithms in 3-dimensions, Master Thesis, Dept. of Computer Science, University of Houston.

[21] C.C. Yao, "New algorithms for bin packing", Journal of Association for Computer Machinery, 27, 1980, pp. 207-227. 